\title{
Teor de Compostos Fenólicos e Atividade Antioxidante do Extrato Seco da Semente de Jabuticaba
}

\author{
Adriana A. de Carvalho, Henrique Páscoa, Frederico S. Martins, \\ Daniela C. Orsi \& Edemilson C. da Conceição
}

O objetivo deste estudo foi determinar as melhores condições para a extração de compostos fenólicos da semente de jabuticaba (Myrciaria cauliflora). O processo de otimização dos parâmetros para a obtenção de extrato líquido da semente de jabuticaba padronizado em teor de compostos fenólicos foi conduzido conforme planejamento Box Behnken (BBD) e Metodologia de Superfície de Respostas (MSR). A capacidade do extrato seco da semente de jabuticaba em sequestrar radicais livres foi medida utilizando o oxidante DPPH. O extrato apresentou maior velocidade cinética que o padrão BHT, atingindo o máximo de consumo do DPPH antes de findar o primeiro minuto da reação, com \%DPPH remanescente menor que 10. Assim, é evidente o elevado potencial do extrato de semente de jabuticaba como substituto do BHT que é amplamente utilizado como antioxidante pela indústria de alimentos.

Palavras-chave: Jabuticaba; compostos fenólicos; atividade antioxidante.

The aim of this study was to determine the best conditions for extraction of phenolic compounds from seed jabuticaba (Myrciaria cauliflora) and optimize the drying parameters to maximize the yield of phenolic compounds in a dry extract. The Box Behnken fatorial design and Response Surface Methodology (RSM) to generate a model that best describe the responses. We obtained the coefficients $\mathrm{R}^{2}$ of 0.97 and 0.92 for the extraction and the drying process, respectively. For the determination of antioxidant was used to test DPPH (2,2-diphenyl-1-picrylhydrazyl). The dry extract showed antioxidant activity above $90 \%$ and proved to be better than the standard BHT used in the food industry. Thus, it is clear the high antioxidant potential of the seed extract jabuticaba.

Keywords: jabuticaba seed; phenolic compounds; antioxidant activity. 


\section{Introdução}

As indústrias de beneficiamento de frutas investem no aumento da sua capacidade de processamento, adquirindo um problema crescente. Ao longo de sua cadeia produtiva, é gerada uma quantidade considerável de resíduos, o que ocasiona desperdícios econômicos e grandes impactos ambientais (SCHIEBER et al., 2001; LOUSADA et al., 2001). Os principais subprodutos que compõem os resíduos agroindustriais do processamento das frutas são a casca, o caroço ou sementes e o bagaço, os quais possuem em sua composição vitaminas, minerais, fibras e compostos antioxidantes importantes para as funções fisiológicas (CONTRERASCALDERON et al., 2011; UCHOA et al., 2008; MATIAS et al., 2005). Estudos têm demonstrado que as frutas são ricas em muitos nutrientes e compostos antioxidantes e que esses constituintes se concentram majoritariamente nas cascas e sementes (GUO et al., 2003, SOONG \& BARLOW, 2004; CONTRERASCALDERON et al., 2011).

A jabuticaba (Myrciaria cauliflora (Mart) O. Berg), fruto nativo do Brasil, possui de 3 a 4 sementes largas (ANDERSEN \& ANDERSEN, 1989) que são descartadas durante o processamento industrial e junto com a casca chegam a representar $50 \%$ do peso do fruto (LIMA et al., 2010). Mesmo sendo popularmente conhecida, poucos são os relatos encontrados na literatura com relação à presença de compostos bioativos na polpa, casca e semente, estando reportada somente a presença de ácido ascórbico, taninos e glicosídeos cianidínicos e peonidínicos (REYNERTSON et al., 2006).

$\mathrm{Na}$ busca por processos produtivos sustentáveis, uma metodologia utilizando extração com solvente e ultrassom foi otimizada para extrair compostos fenólicos da casca da jabuticaba com o mínimo de degradação destes constituintes (SANTOS et al., 2010). A partir dessa otimização, foi elaborado um extrato líquido com alta atividade antioxidante. Todavia, os extratos líquidos são menos estáveis do ponto de vista químico, físico-químico e microbiológico. Neste contexto, destaca-se a importância dos extratos secos que além de apresentar tal estabilidade ainda são mais fáceis de serem padronizados. Além disso, não está ainda descrito procedimento para obtenção de extrato seco da semente de jabuticaba.
Dessamaneira, surge anecessidade derealizar investigação científica e tecnológica que propicie o aproveitamento desse subproduto de forma eficiente, econômica e com o mínimo de degradação dos compostos fenólicos.

\section{Parte Experimental}

\section{OBTENÇÃO E PREPARO DAS AMOSTRAS}

As sementes de jabuticaba foram cedidas pela indústria produtora de fermentados de jabuticaba localizada em Nova Fátima, Goiás, Brasil. Elas foram coletadas diretamente da linha de produção logo após o processo de fermentação. Em seguida, foram transportadas em temperatura ambiente $\left(25^{\circ} \mathrm{C}\right)$ e armazenadas $\left(18{ }^{\circ} \mathrm{C}\right)$ no Laboratório de Química e Bioquímica de Alimentos da Faculdade de Farmácia da UFG. Após serem descongeladas por $12 \mathrm{~h}$ à temperatura de $10^{\circ} \mathrm{C}$, as sementes foram secas a $40^{\circ} \mathrm{C}$ em estufa de circulação de ar (NO 35/5, Solab, São Paulo, Brasil) por cerca de 43h até a redução de $68 \%$ do peso inicial. Em seguida, foram trituradas em micromoinho (Willye TE-648, Tecnal, São Paulo, Brasil) para obtenção de partículas de até $0,5 \mathrm{~mm}$. A amostra triturada foi classificada num conjunto de três peneiras arredondadas, com aberturas de malhas de 150 , 250 e 350 mesh e uma base, de modo que a granulometria das partículas ficassem entre $0,5 \mathrm{~mm}$ e $0,106 \mathrm{~mm}$ retidas na malha de mesh 150; partículas entre $0,105 \mathrm{~mm}$ e 0,067 $\mathrm{mm}$ retidas na malha de mesh 250 e partículas entre 0,066 $\mathrm{mm}$ e $0,045 \mathrm{~mm}$ retidas na malha de mesh 350 .

\section{REAGENTES E PADRÕES}

Foram adquiridos da Sigma-Aldrich Co (St. Louis, MO) os reagentes 2,2-diphenyl-1-picrylhydrazyl $(\mathrm{DPPH} \bullet)$ e Folin-Ciocalteu, o padrão de ácido gálico e o solvente álcool etílico. O padrão 2,6-Di-tertbutyl-4-methylphenol (BHT) foi adquirido da Merck (Hohenbrunn, Germany).

\section{DELINEAMENTO EXPERIMENTAL}

O processo de otimização dos parâmetros para a obtenção de extrato líquido da semente de jabuticaba padronizado em teor de compostos fenólicos foi conduzido conforme planejamento Box Behnken (BBD) e Metodologia de Superfície de Respostas $(\mathrm{MSR})^{13,14}$. Para isso, foram escolhidas três variáveis 
independentes com três níveis e três repetições do ponto central a partir de estudos preliminares. Foram elas: tempo ( $\min , \mathrm{X} 1$ ), abertura da malha da peneira (mesh, $\mathrm{X} 2)$ e teor alcoólico $(\%, \mathrm{X} 3)$. O rendimento da extração dos compostos fenólicos (Y) foi considerado como a resposta (variável dependente) no desenho experimental. Os dados experimentais foram ajustados ao seguinte modelo polinomial de segunda ordem e foram obtidos os coeficientes de regressão:

$$
y=\beta_{0}+\sum_{i=1}^{k} \beta_{i} X_{i}+\sum_{i=1}^{k} \beta_{i i} X_{i}^{2}+\sum_{\substack{i=1 \\ i<j}}^{k-1} \sum_{j=2}^{k} \beta_{i j} X_{i} X_{j}
$$

em que $\beta_{0}, \beta_{\mathrm{i}}, \beta_{\mathrm{ii}}$, e $\beta_{\mathrm{ij}}$, são os coeficientes de regressão para interceptar termos lineares, quadráticos e de interação, respectivamente, e Xi e Xj são as variáveis independentes; $\mathrm{k}$ é o número de variáveis $(\mathrm{k}=3)$. $\mathrm{O}$ modelo determinado foi testado através da Análise de Variância (ANOVA) e teste $t$ dos coeficientes relativos aos seus erros padrões. $O$ software Statistica 7 foi utilizado para gerar a superfície de resposta, mantendo a variável denominada teor de compostos fenólicos (\%) constante no modelo.

Para os procedimentos de secagem, foi utilizado o planejamento estatístico fatorial 2213 . Foram executadas três repetições do ponto central para dar a estimativa do erro experimental. As variáveis independentes utilizadas para avaliar o rendimento do teor de compostos fenólicos (\%), foram: temperatura (min, X1) e o teor de adjuvante $(\%, \mathrm{X} 2)$. O modelo determinado foi testado através da Análise de Variância (ANOVA) e teste $t$ dos coeficientes relativos aos seus erros padrões. O software Statistica 7 foi utilizado para gerar a superfície de resposta, mantendo a variável teor de compostos fenólicos (\%) constante no modelo.

\section{OBTENÇÃO DO EXTRATO LÍQUIDO}

A partir da otimização das condições de extração no planejmanto Box Behnken, foi utilizado o teor alcoólico de $60 \%$ e partículas com granulometira variando entre $0,5 \mathrm{~mm}$ a $0,106 \mathrm{~mm}$ que foram valores mais próximos da sugestão do modelo polinomial para obtenção da maior quantidade possível de compostos fenólicos. Para isso, $1 \mathrm{Kg}$ de pó de semente foi macerado por $24 \mathrm{~h}$ com $2 \mathrm{~L}$ de solvente (60\%). Depois de concluída a maceração, foi iniciado o processo de percolação em percolador de inox com gotejamento de 12 gotas/min. Foram produzidos 10 L de extrato líquido da semente de jabuticaba.

\section{OBTENÇÃO DO EXTRATO SECO}

O extrato recolhido do processo de percolação foi concentrado em equipamento evaporador rotativo (R-220SE, Büchi Labortechnik, AG) a $40{ }^{\circ} \mathrm{C}$ e pressão $65 \mathrm{mBar}$ até conter $5 \%$ sólidos totais, medido por método gravimétrico em analisador com fonte de radiação halógena (Ohaus Inc., Pine Brook, EUA). Cerca de $100 \mathrm{~mL}$ do extrato concentrado foi atomizado em um mini spray dryer (LM MSD1.0, Labmaq do Brasil Ltda, Ribeirao Preto, Brasil) utilizando os parâmetros delineados pelo planejamento (temperatura e teor de adjuvante). Antes de realizar o procedimento de secagem, foi realizada a incorporação da mistura de adjuvante $(60 \%$ de maldodextrina e $40 \%$ de goma arábica) durante 30 min mediante agitação magnética. Em seguida, o spray drier foi ajustado às condições de fluxo de alimentação da amostra de $5 \mathrm{~mL} / \mathrm{min}$, pressão de ar de 4,0 bar, vazão do ar de secagem de 3,0 $\mathrm{m} 3 / \mathrm{min}$, fluxo de ar comprimido a $40 \mathrm{~L} / \mathrm{min}$ e bico injetor com orifício de $1,2 \mathrm{~mm}$ de diâmetro.

\section{DETERMINAÇÃO DO TEOR DE COMPOSTOS FENÓLICOS TOTAIS}

O teor de compostos fenólicos totais foi determinado utilizando o reagente Folin-Ciocalteau (SINGLETON \& ROSSI, 1965) com modificações (HASSIMOTO et. al, 2003). Uma alíquota de $0,25 \mathrm{~mL}$ dos extratos líquidos foi adicionada a $2 \mathrm{~mL}$ de água destilada e $0,25 \mathrm{~mL}$ do reagente de Folin-Ciocalteau. Após três minutos à temperatura ambiente, adicionou-se $0,25 \mathrm{~mL}$ de solução de carbonato de sódio $7,5 \%$ e os tubos foram agitados e levados em banho a $37^{\circ} \mathrm{C}$ durante $30 \mathrm{~min}$. A absorbância foi medida em espectrofotômetro (Femto 700 plus, Femto, São Paulo, Brasil), a $750 \mathrm{~nm}$. O teor de compostos fenólicos foi calculado a partir da equação da reta obtida da curva padrão do ácido gálico:

$$
y=28,18297 x-0,03913\left(R^{2}=0,99977\right) .
$$


Para os extratos secos, foi realizada diluição prévia em uma concentração que permitisse leitura da absorbância dentro da faixa de concentrações do ácido gálico utilizado para a construção da curva. Os resultados foram expressos em miligramas de equivalente de ácido gálico por 100 gramas da amostra (mg EAG/100 g).

\section{DETERMINAÇÃO DA ATIVIDADE ANTIOXIDANTE DO EXTRATO SECO}

A capacidade do extrato seco da semente de jabuticaba em sequestrar radicais livres foi medida utilizando o oxidante DPPH (BRAND-WILLIAMS et al., 1995) com modificações. O extrato foi diluído em metanol na concentração de $0,2 \mathrm{mg} / \mathrm{mL}$. Uma alíquota de $1,5 \mathrm{~mL}$ da solução de DPPH a $20 \mathrm{mg} / \mathrm{L}$ em metanol foi acrescentada a $750 \mu \mathrm{L}$ dos extratos. O potencial antioxidante do extrato foi comparado ao padrão BHT também a $0,2 \mathrm{mg} / \mathrm{mL}$. O decréscimo da absorbância foi monitorado minuto a minuto em espectrofotômetro (Femto 700 plus, Femto, São Paulo, Brasil) durante 20 minutos de reação. $\mathrm{O}$ respectivo branco do extrato e do padrão foram preparados utilizando-se uma alíquota de $750 \mu \mathrm{L}$ do extrato ou da solução de BHT e $1,5 \mathrm{~mL}$ de metanol. A atividade de sequestro do radical DPPH foi calculada pela Equação (1):

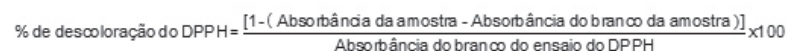

Equação (1)

Para avaliar a cinética da reação entre o extrato e o radical $\mathrm{DPPH}$, foi realizado o monitoramento através do registro da absorbância minuto a minuto até que a reação atingisse um platô de estabilidade. Os percentuais de DPPH remanescentes foram calculados e plotados em função do tempo da reação. Como parâmetro para avaliação da atividade antioxidante, também foi monitorado o comportamento do padrão BHT nas mesmas condições.

\section{MORFOLOGIA DA PARTÍCULA DO EXTRATO SECO}

Amostras do extrato seco foram fixadas na parte superior de uma fita adesiva condutora de carbono, colada em stubs de $12 \mathrm{~mm}$ de diâmetro e $10 \mathrm{~mm}$ de altura. Em seguida, realizou-se a metalização (sputtering) (Sputter
Coater Balzers SCD050, Industry Road, Hatfield) a uma corrente de 40 mTorr durante 180 segundos, tendo como gás de arraste o argônio a um vácuo de 0,05 mbar. As amostras foram observadas em Microscópio Eletrônico de Varredura (Jeol, JSM - 6610, Thermo scientific NSS Spectral Imaging, Waltham, MA). As imagens foram tratadas pelo software Image $1.45 \mathrm{~s}$ e o tamanho das partículas foram determinadas através de medida direta do diâmetro das estruturas esféricas utilizandose a escala das figuras. O diâmetro médio de cada partícula foi estimado com a média entre duas medidas perpendiculares. Também foram tomadas as medidas das esferas de menor e maior diâmetro.

\section{Resultados e Discussão}

\section{OTIMIZAÇÃO DOS PARÂMETROS DE EXTRAÇÃO DE COMPOSTOS FENÓLICOS}

$\mathrm{O}$ delineamento experimental e as respostas para o teor de compostos fenólicos extraídos estão apresentados na Tabela 1. Os coeficientes de regressão de intercessão, linear, quadrático e de interação dos termos do modelo foram calculados usando a técnica dos mínimos quadrados (Tabela 2). Ficou evidente que os coeficientes lineares $X 3$ e os quadráticos X22 e X32 são significativos ao nível de p $<0,05$ e que os coeficientes X3 e X32 são ainda significativos ao nível de $p<0,01$. Os coeficientes das variáveis independentes determinadas para o modelo polinomial de segunda ordem são mostrados na Equação (2):

$\%$ Compostos fenólicos $=-9,27209+0,04881 \mathrm{X} 1+0,01047 \mathrm{X} 2+0,40389 \mathrm{X} 3-$
$0,00106 \mathrm{X} 12-0,00004 \mathrm{X} 22-0,00353 \mathrm{X} 32+0,00009 \mathrm{X} 1 \mathrm{X} 2+0,00011 \mathrm{X} 1 \mathrm{X} 3-0,00001 \mathrm{X} 2 \mathrm{X} 3$

\section{Equação (2)}

Com a análise de variância (Tabela 2), observou-se um $\mathrm{R}^{2}$ de 0,9745 , o que faz com que este seja capaz de explicar $97,45 \%$ do resultado da extração de compostos fenólicos da semente de jabuticaba. Geralmente, a exploração e otimização da metodologia de superfície de resposta pode induzir ao erro, a menos que o modelo apresente um bom ajuste, tornando então a adequação do modelo essencial ${ }^{18}$. Na Figura 1, é possível verificar a interação dos três fatores, variáveis independentes, no rendimento da extração de compostos fenólicos. A 
Tabela 1. Planejamento Box Behnken com três fatores, três níveis, três pontos centrais e resultados experimentais e previstos para extração de compostos fenólicos da semente de jabuticaba (Myrciaria cauliflora).

\begin{tabular}{|c|c|c|c|c|c|c|c|c|c|}
\hline \multicolumn{2}{|c|}{ Experimentos } & \multirow{2}{*}{\multicolumn{2}{|c|}{$\begin{array}{c}\text { Fator } 1\left(X_{1}\right) \\
\begin{array}{c}\text { Tempo } \\
(\min )\end{array}\end{array}$}} & \multirow{2}{*}{\multicolumn{2}{|c|}{$\begin{array}{c}\text { Fator } 2\left(X_{2}\right) \\
\text { Abestura da } \\
\text { peneina (mesh) }\end{array}$}} & \multirow{2}{*}{\multicolumn{2}{|c|}{$\begin{array}{c}\text { Fator } 3\left(X_{3}\right) \\
\begin{array}{c}\text { Teor alcoúlico } \\
(\mathbf{X})\end{array}\end{array}$}} & \multicolumn{2}{|c|}{ 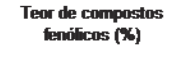 } \\
\hline \multirow{2}{*}{$\begin{array}{l}\text { Ondem } \\
\text { padrä } \\
1\end{array}$} & \multirow{2}{*}{$\begin{array}{c}\begin{array}{c}\text { Ondem de } \\
\text { execuçäâ }\end{array} \\
10\end{array}$} & & & & & & & Previsto & Experimental \\
\hline & & 15 & $(-1)$ & 150 & $(-1)$ & 60 & (0) & 3,67 & 3,80 \\
\hline 2 & 6 & 45 & $(+1)$ & 150 & $(-1)$ & 60 & (0) & 3,85 & 4,10 \\
\hline 3 & 8 & 15 & $(-1)$ & 350 & $(+1)$ & 60 & (0) & 1,93 & 1,66 \\
\hline 4 & 1 & 45 & $(+1)$ & 350 & $(+1)$ & 60 & (0) & 2,67 & 2,52 \\
\hline 5 & 7 & 15 & $(-1)$ & 250 & (0) & 40 & $(-1)$ & 2,15 & 2,25 \\
\hline 6 & 5 & 45 & $(+1)$ & 250 & (0) & 40 & $(-1)$ & 2,61 & 2,53 \\
\hline 7 & 3 & 15 & $(-1)$ & 250 & (D) & 80 & $(+1)$ & 1,43 & 1,43 \\
\hline 8 & 4 & 45 & $(+1)$ & 250 & (0) & 80 & $(+1)$ & 1,89 & 1,84 \\
\hline 9 & 15 & 30 & (0) & 150 & $(-1)$ & 40 & $(-1)$ & 2,95 & 2,71 \\
\hline 10 & 14 & 30 & (0) & 350 & $(+1)$ & 40 & $(-1)$ & 1,49 & 1,68 \\
\hline 11 & 9 & 30 & (0) & 1500 & $(-1)$ & 80 & (+1) & 2,23 & 2,04 \\
\hline 12 & 12 & 30 & (0) & 350 & $(+1)$ & 80 & $(+1)$ & $0, \pi 7$ & 0,96 \\
\hline 13 & 11 & 30 & (0) & 250 & (0) & 60 & (0) & 3,67 & 3,68 \\
\hline 14 & 13 & 30 & (0) & 250 & (D) & 60 & (0) & 3,67 & 3,66 \\
\hline 15 & 2 & 30 & (0) & 250 & (0) & 60 & (0) & 3,67 & 3,66 \\
\hline
\end{tabular}

Tabela 2. Coeficientes de regressão para o modelo polinomial quadrático e análise de variância (ANOVA) para os resultados experimentais da extração de compostos fenólicos da semente de jabuticaba (Myrciaria cauliflora)

\begin{tabular}{|c|c|c|c|}
\hline Modelo & $\begin{array}{c}\text { Coeficiente de } \\
\text { requessäo }\end{array}$ & $\underset{\text { padräo }}{\text { Emo }}$ & p \\
\hline & $-9,27209$ & 2,183400 & 0,0198118 \\
\hline \multicolumn{4}{|l|}{ Linear } \\
\hline$x_{1}$ & 0,04881 & 0,051001 & 0,382543 \\
\hline$x_{2}$ & 0,01047 & 0,018473 & 0,271486 \\
\hline$x_{3}$ & 0,407899 & 0,046907 & 0,0100349 \\
\hline \multicolumn{4}{|l|}{ Quadrático } \\
\hline$x_{1}^{2}$ & $-0,00106$ & 0,000615 & 0,145469 \\
\hline$x_{2}^{2}$ & $-0,00004$ & 0,0010014 & 0,032967 \\
\hline$x_{3}^{2}$ & $-0,00353$ & 0,000346 & 0,000155 \\
\hline \multicolumn{4}{|l|}{ hteraçāo } \\
\hline$x_{1} x_{2}$ & 0,00009 & 0,0101899 & 0,339072 \\
\hline$x_{1} x_{3}$ & 0,00011 & 0,0001443 & 0,8007599 \\
\hline$x_{2} x_{3}$ & - 0,00:01 & 0,0010657 & 0,932845 \\
\hline $\mathbf{R}^{2}$ & 0,97455 & & \\
\hline
\end{tabular}

partir do modelo gerado é possível atingir o máximo da extração de compostos fenólicos utilizando os seguintes valores para a combinação: $33 \min (\mathrm{X} 1=$ tempo $), 163$ mesh (X2 = abertura da malha da peneira) e $57 \%$ (X3 = graduação alcoólica).
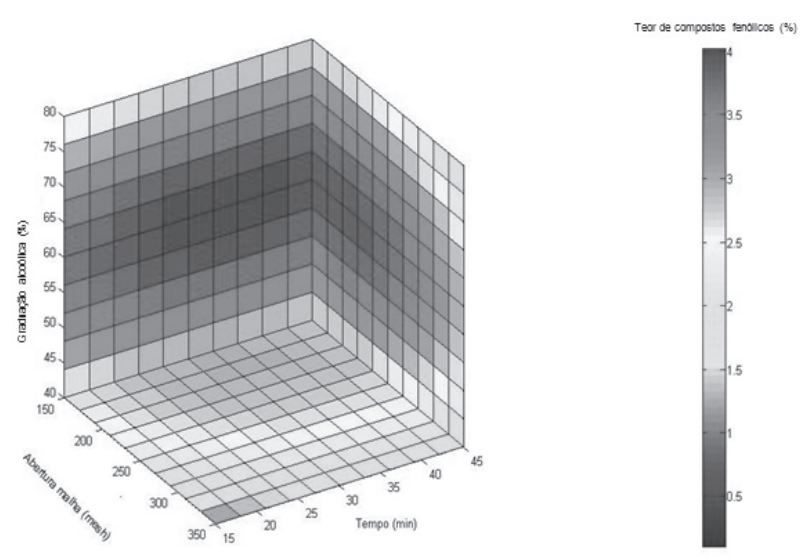

Figura 1. Superfície de resposta demonstrando o efeito do tempo de extração (min), granulometria das partículas retidas na malha (mesh) e graduação alcoólica (\%) no teor de compostos fenólicos (\%).

\section{OTIMIZAÇÃO DOS PARÂMETROS DE SECAGEM DO EXTRATO}

Os dados da Tabela 3 apresentam o delineamento experimental e as respostas para o rendimento compostos fenólicos no produto final. Os coeficientes de regressão de intercessão, linear e de interação dos termos do modelo foram calculados usando a técnica dos mínimos quadrados e são apresentados na Tabela 4. Os coeficientes das variáveis independentes determinadas para o modelo são mostrados na Equação (3) de primeira ordem:

\% Compostos Fenólicos $=35,95992-0,012178 * X 1+$
$0,052938 *$ X2 $-0,0000903383$ X1X2 Equação (3)

A análise de variância (Tabela 4) apresentou um $\mathrm{R}^{2}$ de 0,9203 , o que faz com que este seja capaz de explicar $92,03 \%$ do resultado do rendimento do teor de compostos fenólicos no extrato seco da semente de jabuticaba. Apesar do coeficiente $\mathrm{R}^{2}$ ser elevado, nenhuma das variáveis independentes foi significativa ao nível de $\mathrm{p}<0,05$ apenas o coeficiente de intercessão. 
Tabela 3. Planejamento fatorial completo $2^{2}$ com três pontos centrais e resultados experimentais e previstos para rendimento de compostos fenólicos no extrato seco da semente de jabuticaba (Myrciaria cauliflora).

\begin{tabular}{|c|c|c|c|c|c|}
\hline \multicolumn{2}{|c|}{ Experimentos } & \multirow{2}{*}{$\begin{array}{l}\text { Falor } 1\left(X_{1}\right) \\
\text { Temperalua } \\
\text { ('C) }\end{array}$} & \multirow{2}{*}{$\begin{array}{c}\text { Fator } 2\left(X_{2}\right) \\
\text { Adfwante } \\
\text { (X) }\end{array}$} & \multicolumn{2}{|c|}{$\begin{array}{l}\text { Teor de compostos } \\
\text { fenólicos ( } \mathrm{x})\end{array}$} \\
\hline $\begin{array}{l}\text { Ondem } \\
\text { padräo }^{B}\end{array}$ & $\begin{array}{l}\text { Oudem de } \\
\text { execuçäำ }\end{array}$ & & & Prenisto & Experimental \\
\hline 1 & 7 & $80(-1)$ & $5(-1)$ & 35,92 & 35,21 \\
\hline 2 & 4 & 120 (1) & $5(-1)$ & 35,89 & 34,71 \\
\hline 3 & 6 & 80 (-1) & 20 (1) & 35,03 & 35,90 \\
\hline 4 & 1 & 120 (1) & 20 (1) & 36,00 & 35,34 \\
\hline 5 & 5 & 100 (ㅁ) & 12,5 (ㅁ) & 35,96 & 33,21 \\
\hline 6 & 3 & $100(0)$ & $12,5(0)$ & 35,95 & 34,07 \\
\hline 7 & 2 & 100 (마) & 12,5 (0) & 35,96 & 33,11 \\
\hline
\end{tabular}

Tabela 4. Coeficientes de regressão para o modelo linear e análise de variância (ANOVA) para os resultados experimentais da secagem do extrato da semente de jabuticaba (Myrciaria cauliflora).

\begin{tabular}{|c|c|c|c|}
\hline Modelo & $\begin{array}{c}\text { Coeficiente de } \\
\text { regressâa }\end{array}$ & Emo & $p$ \\
\hline & 35,95992 & 2,611788 & 0,0155234 \\
\hline \multicolumn{4}{|l|}{ Linear } \\
\hline$x_{1}$ & $-0,01218$ & 0,025611 & 0,681296 \\
\hline$x_{2}$ & 0,05294 & 0,179167 & 0,795488 \\
\hline \multicolumn{4}{|l|}{ Iteraçลี่ } \\
\hline$x_{1} x_{2}$ & -0,010119 & 0,001757 & 0,963965 \\
\hline $\mathbf{R}^{2}$ & 0,92039 & & \\
\hline
\end{tabular}

\section{ATIVIDADE ANTIOXIDANTE DO EXTRATO SECO}

O comportamento cinético da reação do DPPH com o extrato seco da semente de jabuticaba e o padrão BHT, na concentração de $0,2 \mathrm{mg} / \mathrm{mL}$, é mostrado na Figura 2, através da curva relativa ao decréscimo da porcentagem de DPPH remanescente em função do tempo (min). É possível observar que o extrato apresentou maior velocidade cinética que o padrão BHT, atingindo o máximo de consumo do DPPH antes de findar o primeiro minuto da reação, com \% DPPH remanescente menor que 10. Dessa maneira, tendo em vista que o tempo de reação é um parâmetro importante na avaliação da capacidade antioxidante (SANCHEZ-MORENO et al., 1998), é possível afirmar que o grupo de compostos fenólicos presentes no extrato seco da semente de jabuticaba foi mais eficiente que o padrão BHT. Assim fica evidenciado que o extrato seco da semente de jabuticaba exibe um forte potencial antioxidante e que os parâmetros utilizados no ensaio de secagem com o maior rendimento de compostos fenólicos não foi capaz de interferir em sua atividade.

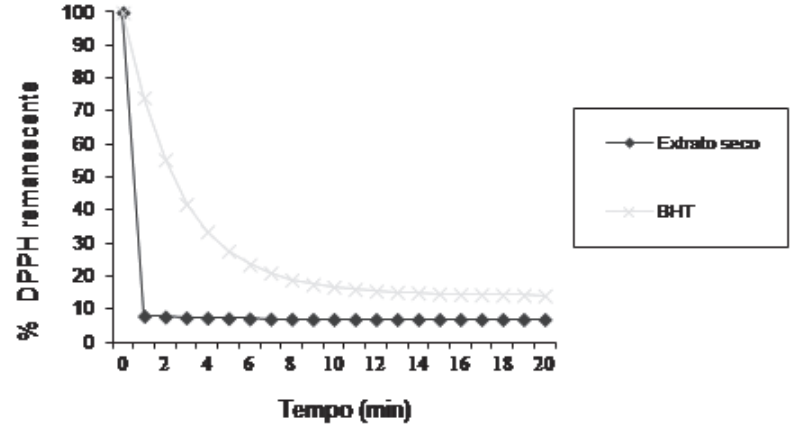

Figura 2. Comparação do comportamento cinético do extrato seco de jabuticaba (Myrciaria cauliflora) e do padrão BHT frente ao radical DPPH.

\section{EFEITO DAS CONDIÇÕES DE SECAGEM SOBRE A MICROESTRUTURA DA PARTÍCULA DO EXTRATO SECO}

O extrato seco da semente de jabuticaba (Figuras 3 e 4) apresentou partículas de tamanhos diferenciados. Todavia, existiu uma maior frequência $(80 \%)$ para o tamanho de $1 \mu \mathrm{m}$ (Figura 5). As micropartículas podem ter diâmetros variando entre unidades de micrometros até milímetros e apresentar diversas formas, dependendo dos materiais e métodos usados para sua obtenção. Essas partículas são classificadas de acordo com o seu tamanho em nanopartículas $(0,01$ a $0,2 \mu \mathrm{m})$ ou em micropartículas (1 a $100 \mu \mathrm{m})$ (MARTIN, 1993). Dessa maneira, as partículas produzidas neste experimento podem ser consideradas como micropartículas. Avaliando ainda as imagens (Figuras 3 e 4), é possível constatar a presença de algumas microesferas de aspecto rugoso. Se o material de parede forma microcápsulas livres de qualquer deformação, maior será a capacidade deste material em reter e proteger o material ativo, neste caso, os compostos fenólicos (ROSENBERG et al. 1985). A formação de microestruturas com superfície rugosa, característica indesejável, pode afetar as condições de escoamento do material (ROSENBERG et al. 1990). As imperfeições superficiais ocorrem quando o processo de formação de filme durante a secagem das gotículas atomizadas é lento (RE, 1998). Apesar do aumento da temperatura de secagem, por sua vez, o aumento da taxa de formação de filme na superfície da gota evita o aspecto rugoso (RE, 1998), neste estudo ocorreu o contrário. Há 


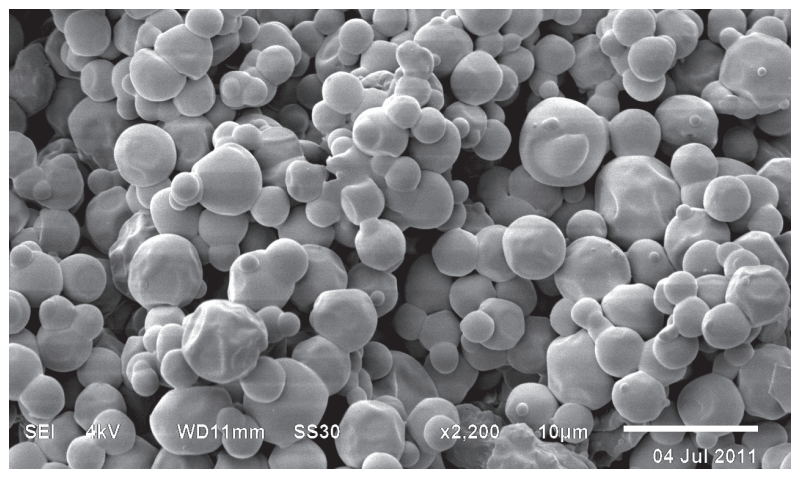

Figura 3. Fotomicrografia obtida por microscopia eletrônica de varredura (MEV) do extrato seco de semente de jabuticaba (Myrciaria cauliflora) por processo de secagem em spray dryer, cujas condições foram: Temperatura $72^{\circ} \mathrm{C}$ e Adjuvante $12,5 \%$. SEI: Signal; $4 \mathrm{kV}=\mathrm{Acc}$ Voltage; SS35 = Spotsize; x2200 = aumento.

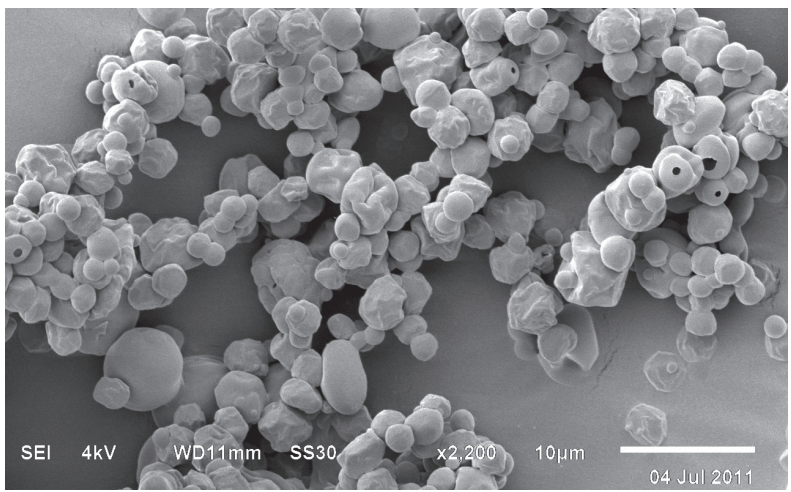

Figura 4. Fotomicrografia obtida por microscopia eletrônica de varredura (MEV) do extrato seco de semente de jabuticaba (Myrciaria cauliflora) por processo de secagem em spray dryer, cujas condições foram: Temperatura $128^{\circ} \mathrm{C}$ e Adjuvante $12,5 \%$. SEI: Signal; $4 \mathrm{kV}=\mathrm{Acc}$ Voltage; SS25 = Spotsize; x2200 = aumento.

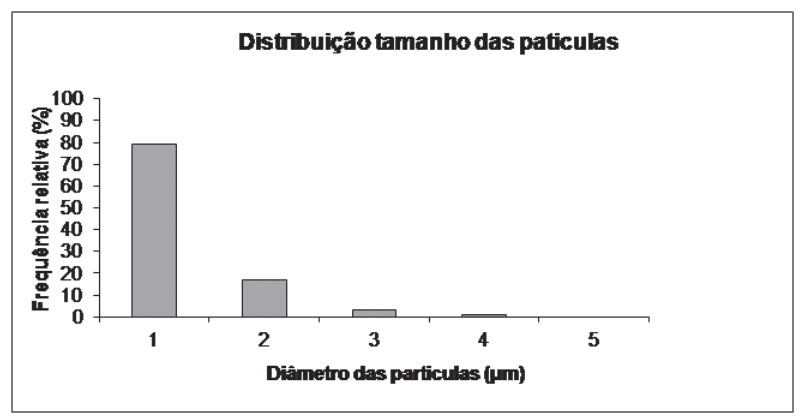

Figura 5. Distribuição do tamanho das partículas do extrato seco da semente de jabuticaba (Myrciaria cauliflora). maior predominância de microesferas de superfície lisa no ensaio com temperatura de $72^{\circ} \mathrm{C}$ (Figura 3). Dessa maneira, o sucesso na formação de microesferas de superfície lisa dependerá dos parâmetros de processo e da concentração da mistura e do tipo de adjuvante utilizado no sistema (ASCHERI et al. 2003) e não somente da temperatura do processo.

\section{Conclusão}

O alto teor de compostos fenólicos e sua respectiva atividade antioxidante observado no extrato seco da semente de jabuticaba sugerem que as sementes podem ser uma boa fonte de compostos bioativos. Além disso, a obtenção deste extrato poderá propiciar várias aplicações industriais, tendo em vista que este mostrou ser mais eficiente que o BHT. Adicionalmente, este extrato também poderá ser utilizado na suplementação de dietas e na formulação de novos alimentos com alegações de propriedades funcionais. Também, a sua produção seria uma alternativa para diminuir os impactos ambientais gerados pelo descarte de resíduos agroindustriais no solo.

\section{Agradecimento}

Os autores agradecem à Fazenda Jaboticabal pela doação das amostras de semente de jabuticaba, à CAPES/CNPq pela concessão da bolsa de estudos e ao Laboratório Multiusuário de Microscopia de Alta Resolução (LABMIC) do Instituto de Física da UFG pela realização das fotomicrografias.

\section{Referência}

1. Ascheri, D. P. R.; Marquez, M. O. M.; Martucci, E. T. Microencapsulação de óleo essencial de laranja: seleção de material de parede. Ciência e Tecnologia de Alimentos, Campinas, v.23, p. 1-6, 2003.

2. Andersen, O.; Andersen, V. U. As frutas silvestres brasileiras. São Paulo: Globo, 1989. 203 p.

3. Contreras-Calderón, J.; Calderón-Jaimes, L.; Guerra-Hernández, E.; García-Villanova, B. Antioxidant capacity, phenolic content and vitamin $\mathrm{C}$ in pulp, peel and seed from 24 exotic fruits from Colombia. Food Research International, Barking, v. 44, p. 2047-2053, 2011.

4. Barros Neto, B.; Scarminio, I. S.; Bruns, R. E.; Como fazer experimentos: pesquisa e desenvolvimento na ciência e na indústria, 2a ed., Ed. Unicamp: Campinas, 2002. 
5. Box, G. E. P.; Hunter, W. G.; Hunter, J. S.; Statistic for Experimenters: an Introduction to design, data analysis and model building. Wiley: New York, 1978.

6. Brand-Williams, W.; Cuvelier, M.; Berset, C. Use of a Free Radical Method to Evaluate Antioxidant Activity. Journal of Food Science and Technology, Mysore, v. 28, n. 1, p. 25-30, 1995.

7. Guo, C.; Yang, J.; Wei, J.; Li, Y.; Xu, J.; Jiang, Y. Antioxidant activities of peel, pulp and seed fractions of common fruits as determined by FRAP assay. Nutrition Research, Tarrytown, v. 23, n. 12 , p. $1719-1726,2003$

8. Hassimoto, N. M. A.; Genovese, M. I.; Santos, R. J.; Lajolo, F. M. Determinação do conteúdo de fenólicos totais em frutas. Revista de Ciências Farmacêuticas, Araraquara, v.39, 167-169, 2003.

9. Lima, A. J. B., Corrêa, A.D., Alves, A. P. C., Abreu, C. M. P., \& Dantas-Barros, A. M. Caracterização química do fruto jabuticaba (Myrciaria cauliflora Berg) e de suas frações. Archivos Latinoamericanos de Nutricíon, Caracas, v. 58, p. 416-421, 2008.

10. Lousada Junior, J. E.; Neiva, J. N. N.; Rodriguez, N. M.; Pimentel, J. C. M. P.; Lôbo, R. N. B. Consumo e digestibilidade de subprodutos do processamento de frutas em ovinos. Revista Brasileira de Zootecnia, Viçosa, v.34, n.2, p.659-669, 2005.

11. Martin, A. N.; Physical pharmacy: Physical chemical principles in the pharmaceutical sciences, Ed. Lea \& Febiger: USA, 1993.

12. Matias, M.F.O.; Oliveira, E.L.; Gertrudes, E.; Magalhâes, M.A. Use of fibres obtained from the cashew (Anacardium ocidentale, L) and guava (Psidium guayava) fruits for enrichment of food products. Brazilian Archives of Biology and Technology, Curitiba, v.48, p.143-150, 2005

13. RE, M. I. Microencapsulation by spray drying. Drying technology, v.16, n.6, p. 1195-1236, 1998.

14. Reynertson, K. A.; Wallace, A. M.; Adachi, S.; Gil, R. R; Yang, H.; Basile, M. J. Bioactive depsides anal anthocyanins from Jaboticaba (Myrciaria caulifora). Journal Natural Products, Washington, v. 69, p. 1228-1230, 2006.

15. Rosenberg, M.; Kopelman, I. J.; Talmon, Y.; Factors affecting retention in spray-drying microencapsulation of volatile materials. Journal of Agriculture and Food Chemistry, v. 38, n, 5, p. 12881294,1990

16. Rosenberg, M., Kopelman, I. J.; Talmon, Y., A study of microencapsulation by scanning electron microscopy. Journal of Food Sciense, v.50, p. 139-144, 1985.

17. Sanchez-Moreno, C.; Larrauri, J. A.; Saura-Calixto, F. A procedure to measure the antiradical efficiency of polyphenols. Journal of the Science of Food and Agriculture, v. 76, 270-276, 1998.

18. Santos, D. T., Veggi, P. C., M. Angela A. Meireles. Extraction of antioxidant compounds from jabuticaba (Myrciaria cauliflora) skins: yield, composition and economical evaluation. Journal of Food Engineering, London, v. 101, p. 23-31, 2010.

19. Singleton, V. L.; ROSSI, J. A. Colorimetry of total phenolics with phosphomolybdic-phosphotungstic acid reagents. American Journal of Enology and Viticulture, Davis, v. 16, n. 3, p. 144-158, 1965.

20. Schieber, A., Stintzing, F. C., Carle, R. By-products of plant food processing as a source of functional compounds - recent developments. Trends in Food Science and Technology, Cambridge, v. 12, p. 401-413, 2001.

21. Soong, Y. Y.; Barlow, P. J. Antioxidant activity and phenolic content of selected fruit seeds. Food Chemistry, Washington, v. 88, n. 3, p. 411-417, 2004.

22. Uchôa, A. M. A.; Costa, J. M. C.; Maia, G. A.; Silva, E. M. C.; Carvalho, A. F. F. U.; MeirA, T. R. Parâmetros físico-químicos, teor de fibra bruta e alimentar de pós alimentícios obtidos de resíduos de frutas tropicais. Segurança Alimentar e Nutricional, Campinas, v.15, n.2, p.58-65, 2008.

23. Toneli, J. T. C. L.; Park, K. J.; Murr, F. E. X.; Negreiros, A. A. Efeito da umidade sobre a microestrutura da inulina em pó. Ciência e Tecnologia de Alimentos, Campinas, v. 28, n.1, p. 122126, 2008.

\section{Adriana A. de Carvalho ${ }^{\text {*, }}$ Henrique Páscoa ${ }^{2}$, Frederico S. Martins ${ }^{2}$, Daniela C. Orsi ${ }^{2}$ \& Edemilson C. da Conceição ${ }^{2}$}

${ }^{1}$ Instituto SENAI de Tecnologia em Alimentos e Bebidas, Rua Professor Lázaro Costa $n^{\circ}$ 348, Vila Canaã, CEP: 74415-420, Goiânia,Goiás, Brasil.

${ }^{2}$ Laboratório de PD\&I de Fitoterápicos, Fitocosméticos e Nutracêuticos, Faculdade de Farmácia da Universidade Federal de Goiás, Av. Universitária com $1^{\text {a }}$ Avenida s/n, Setor Universitário, CEP: 74605-220, Goiânia, Goiás, Brasil

*Email: adrianaantunes.senai@sistemafieg.org.br 